\title{
Control of mirror segment actuators for the European Extremely Large Telescope
}

Gert Witvoet, Remco den Breeje, Jan Nijenhuis, and Niek Doelman

Controller design based on dynamic analysis proves compliant performance of highly accurate actuators for piston-tip-tilt correction of primary mirror segments for the European Extremely Large Telescope.

The primary mirror (M1) of the European Extremely Large Telescope (E-ELT) will consist of 798 mirror segments, which together form a smooth $39.3 \mathrm{~m}$ optical surface. To achieve optimal performance in such a segmented concept, ${ }^{1}$ each individual mirror segment must be actively controlled in piston, tip, and tilt, using three dedicated single-degree-of-freedom (DOF) actuators. These PACTs (Position ACTuators) need to combine nanometer-level accuracy with a centimeter-range stroke, while carrying a large mass in a changing gravitational field and being exposed to nonstationary structural vibrations and wind loads. In collaboration with VDL Enabling Technologies Group, we have designed and realized various different PACT prototypes ${ }^{2}$ for the E-ELT. Here, we will present some of the analysis and control results ${ }^{3}$ we have obtained with one of the most recent prototypes, proving compliance with the E-ELT requirements. ${ }^{4}$

The E-ELT application requires that an actuator achieve $1.7 \mathrm{~nm}$ rms position accuracy in the presence of vibrations and wind disturbances, while tracking a $15 \mathrm{~mm}$ stroke with a velocity up to $1.2 \mu \mathrm{m} / \mathrm{s}$. Due to the large ratio between stroke and accuracy $\left(10^{7}\right)$, our actuator uses a two-stage concept, with a voice coil for accuracy and a DC motor for off-loading and large stroke. Moreover, it has an on-axis nanometer-resolution feedback sensor, and an internal spring to carry the segment weight (up to $900 \mathrm{~N}$ per actuator). Figure 1 shows an image of a specific prototype.

The dynamic behavior of the actuator has been assessed in a dedicated E-ELT relevant test setup, in which the PACT has been tested in combination with a $90 \mathrm{~kg}$ mass under various orientations. Figure 2 shows a large number of frequency

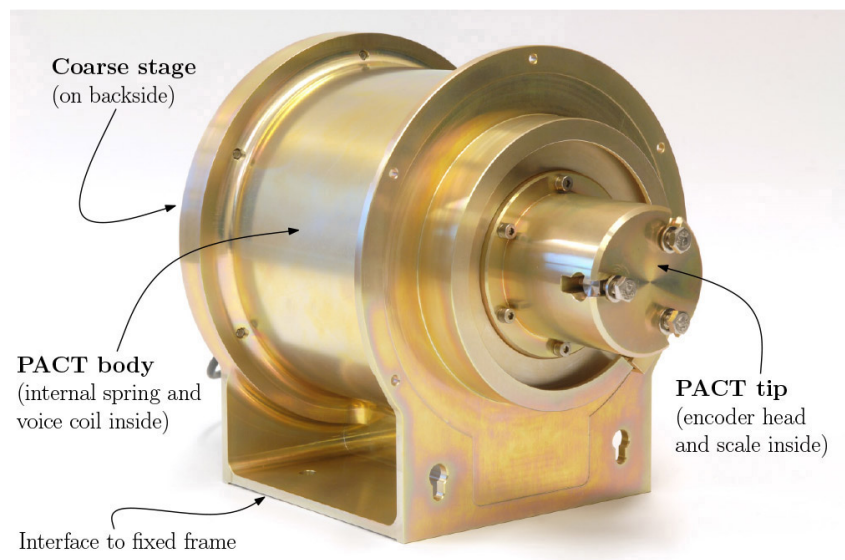

Figure 1. Picture of one of the recent PACT (Position ACTuator) prototypes. Voice coil, internal spring, and feedback sensor are integrated inside the PACT body. The coarse stage is mounted at the back side. (Photo: TNO/Fred Kamphues.)

response function (FRF) measurements, ${ }^{5}$ each taken at a different nominal point along the full stroke of the actuator (in a certain orientation). These FRFs show that the dynamics are very clean up to about $300 \mathrm{~Hz}$ - i.e., there are no spurious modes in this frequency range-and the dynamics are independent of the actuator stroke. This is very beneficial from a control point of view, as it enables a robust high-bandwidth controller design.

Comparison with an accurate analytic model of the PACT in its test setup has given detailed insight in the mode shapes of the system. At $11.1 \mathrm{~Hz}$, the total moving mass resonates on the PACT internal springs, whereas the anti-resonance-resonance pair at 60 and $400 \mathrm{~Hz}$ corresponds to the decoupling of the $90 \mathrm{~kg}$ mass from the moving mass of PACT $(1.32 \mathrm{~kg})$. The small modes just below $10 \mathrm{~Hz}$ are suspension modes of the test setup itself.

Continued on next page 
The architecture presented in Figure 3 has been proposed to control the actuator. The upper loop represents the feedback loop on the fine stage, where the voice coil force $F_{\mathrm{VC}}$ is used to achieve high accuracy on the PACT position $y$. Using loopshaping techniques, ${ }^{6}$ a high-bandwidth $(85 \mathrm{~Hz})$ controller $C_{\mathrm{VC}}$ has been designed and specifically tuned on the fine-stage system dynamics $H_{\mathrm{VC}}$ (represented by the FRF measurements in Figure 2) and information on the external vibrations and wind disturbances. This controller consists of a double integrator, lead filter, low-pass filter, and two inverse notches, and is robustly stable over the full actuator stroke.

When the actuator is tracking a continuously increasing (or decreasing) reference $y_{\text {ref }}$, the lower loop in Figure 3 is needed to off-load the voice coil. This coarse stage loop controls the voice coil force toward zero using the velocity of the DC motor $v_{\mathrm{DC}}$. The bandwidth of this loop can, however, be very low (well below $1 \mathrm{~Hz}$ ), so that the controller $C_{\mathrm{DC}}$ is a simple gain with a low-pass filter.

The designed controllers have been implemented in real time, with which the actuator has been subjected to an E-ELT-relevant tracking scenario. The position set point is changed with a tracking velocity of $1.2 \mu \mathrm{m} / \mathrm{s}$ in both the positive and negative directions, thereby covering more than $500 \mu \mathrm{m}$ of stroke, while the test setup is subjected to ground vibrations and simulated wind disturbances. Figure 4 depicts the resulting closed-loop response, as measured by the internal metrology. This plot shows that the set point is accurately tracked within roughly $\pm 10 \mathrm{~nm}$ error during the complete experiment. The error is marginally larger in the backward 'downhill' motion than in the for-

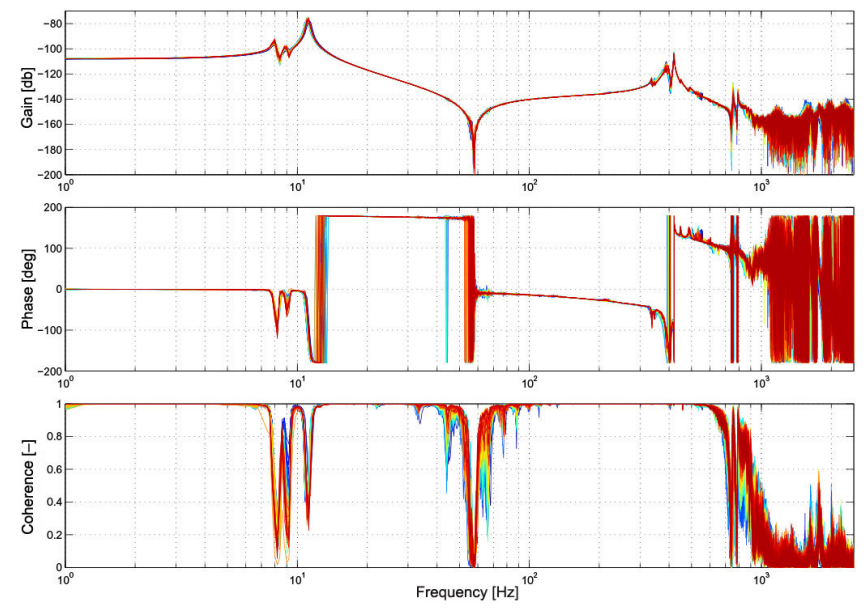

Figure 2. Measured frequency response functions (FRFs) of the PACT in its test setup, as a function of the coarse stage position. Dark blue is fully retracted. Dark red is fully extracted.

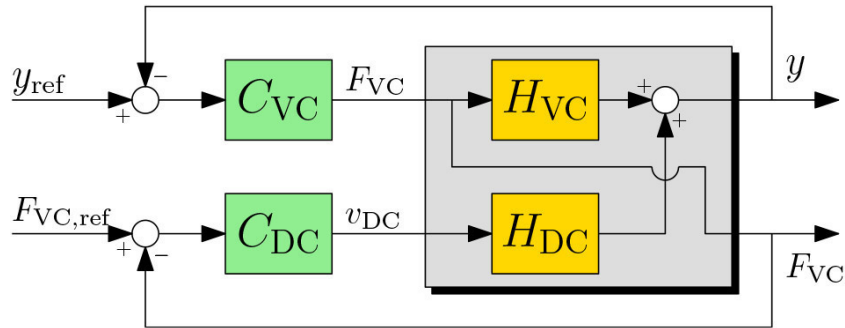

Figure 3. Block diagram of the combined fine and coarse stage control architecture. $y_{\text {ref }}$ : Reference. F: Force. C: Controller. H: System dynamics. DC: DC motor. $v_{D C}$ : Velocity of the DC motor. VC: Voice coil.
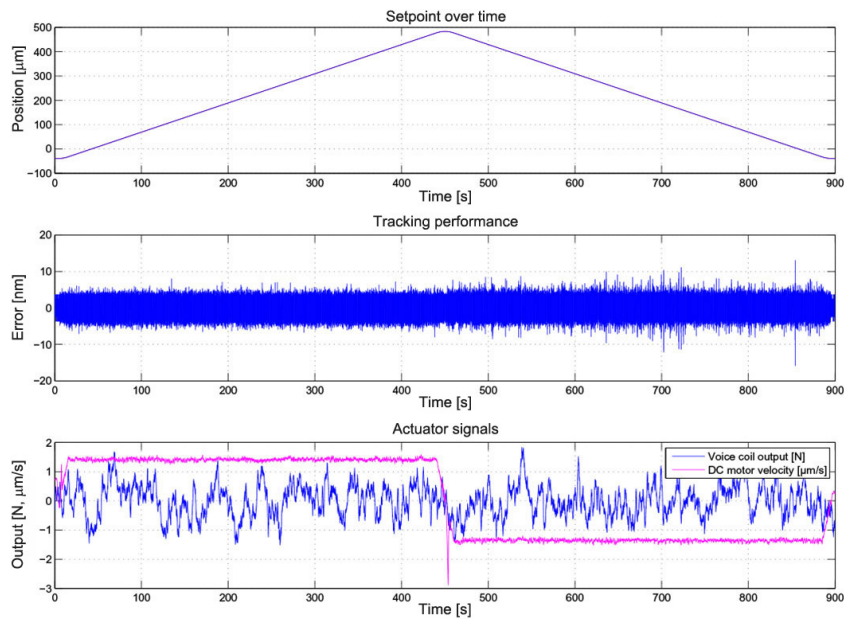

Figure 4. Measured closed-loop tracking results: setpoint and output (top), tracking error (middle), and voice coil force and DC-motor velocity (bottom).

ward 'uphill' motion, which can be attributed to the self-locking behavior of the coarse stage. The impact on the fine stage performance is, however, very small. Overall, the error sums up to just $1.4 \mathrm{~nm}$ rms, i.e., well within specifications.

The voice coil force in the bottom plot clearly shows the compensation of the low-frequent wind disturbances. Still, this force nicely oscillates around zero, well within $\pm 2 \mathrm{~N}$, owing to the DC-motor off-loading with nearly constant velocity. In this experiment the voice coil power consumption sums to just $7.6 \mathrm{~mW}$ rms, with an instantaneous peak of $54 \mathrm{~mW}$.

In summary, we have demonstrated that the performance of the our PACT prototypes is compliant with the challenging E-ELT requirements. Moreover, we have presented a robust analysis and controller design approach to do so. Future work

Continued on next page 
will include interaction analyses of three PACTs underneath a mirror segment and advanced multiple-input-multiple-output controller design to achieve optimal performance at mirror segment level.

\section{Author Information}

\section{Gert Witvoet, Remco den Breeje, Jan Nijenhuis, and} Niek Doelman

$\mathrm{TNO}$

Delft, The Netherlands

Gert Witvoet is a control specialist at TNO Technical Sciences, working on space, astronomy, semiconductor, and nuclear fusion applications. In 2011 he received his $\mathrm{PhD}$ in mechanical engineering, with a specialization in control engineering, from the Eindhoven University of Technology. He is also a lecturer on motion control at the same university.

Remco den Breeje received his BSc in applied physics from the The Hague University of Applied Sciences in 2005. At TNO he has specialized in real-time control platforms and algorithms. Specific application domains include adaptive optics, mechatronics, and space instruments.

Jan Nijenhuis works as systems engineer at TNO Technical Sciences. In 1980 he graduated from the Delft University of Technology in structural engineering and design. He has specialized in optomechanical system design, for which he received the Rien Koster Award from the Dutch Society for Precision Engineering in 2008.

Niek Doelman received his $\mathrm{PhD}$ in applied physics from the Delft University of Technology in 1993. He is a senior scientist at TNO working on adaptive optics, vibration control, and mechatronics, and-since 2012 - a part-time professor in Control for Astronomy Instruments at the Leiden Observatory.
References

1. R. C. Jared, A. A. Arthur, S. Andreae, A. K. Biocca, R. W. Cohen, J. M. Fuertes, J. Franck, et al., The W. M. Keck Telescope segmented primary mirror active control system, Proc. SPIE 1236, p. 996, 1990. doi:10.1117/12.19266

2. F. Kamphues, J. R. Nijenhuis, R. den Breeje, T. C. van den Dool, and J. Ponsioen, PACT: the actuator to support the primary mirror of the ELT, Proc. SPIE 7018, p. 70180Z, 2008. doi:10.1117/12.789921

3. G. Witvoet, R. den Breeje, J. Nijenhuis, R. Hazelebach, and N. Doelman, Dynamic analysis and control of mirror segment actuators for the European Extremely Large Telescope, J. Astron. Telescopes Instr. Syst. 1 (1), p. 019003, 2015. doi:10.1117/1.JATIS.1.1.019003

4. T. Erm, Technical specification for the manufacturing and delivery of prototype position actuators for the primary mirror of the European Extremely Large Telescope (E-ELT) Tech. Rep. E-SPE-ESO-142-0011, Issue 2, ESO, 2009.

5. R. Pintelon and J. Schoukens, System Identification: A Frequency Domain Approach, Wiley-IEEE Press, 2004

6. S. Skogestad and I. Postlethwaite, Multivariable Feedback Control: Analysis and Design, 2nd ed., Wiley, 2005. 